\title{
The Role of Hepatic Arterial Infusion Chemotherapy in the Treatment of Hepatocellular Carcinoma: A Systematic Review and Meta-Analysis
}

\author{
Shengzhou $\mathrm{Li}^{\mathrm{a}}$ Jiaxuan Xu ${ }^{\mathrm{b}}$ Hongya Zhang ${ }^{\mathrm{c}}$ Jiaze Hong ${ }^{\mathrm{b}}$ Yuexiu Si ${ }^{\mathrm{d}}$ \\ Tong Yang ${ }^{e}$ Yujing $\mathrm{He}^{\mathrm{b}}$ Derry Minyao $\mathrm{Ng}^{f}$ Dingcheng Zheng ${ }^{g}$

\begin{abstract}
${ }^{a}$ Emergency Medical Center, Ningbo Yinzhou No. 2 Hospital, Ningbo, China; ${ }^{\text {b}}$ The Second Clinical Medical College, Zhejiang Chinese Medical University, Hangzhou, China; 'Thyroid and Breast Surgery, Ningbo Yinzhou No. 2 Hospital, Ningbo, China; ${ }^{d}$ School of Basic Medical Sciences, Zhejiang Chinese Medical University, Hangzhou, China; ${ }^{\text {eDepartment }}$ of Tumor HIFU Therapy, HwaMei Hospital, University of Chinese Academy of Sciences, Ningbo, China; ${ }^{\mathrm{f}}$ Medical College of Ningbo University, Ningbo, China; 'Department of General Surgery, HwaMei Hospital, University of Chinese Academy of Sciences, Ningbo, China
\end{abstract}

\section{Keywords}

Hepatocellular carcinoma $\cdot$ Hepatic arterial infusion

chemotherapy · Meta-analysis

\begin{abstract}
Background: The main aim of this study was to investigate comprehensively the clinical effect of hepatic arterial infusion chemotherapy (HAIC) on patients suffering from hepatocellular carcinoma (HCC). Methods: The following electronic databases were searched for eligible articles published from inception to July 2020: PubMed, Web of Science, Embase, and Cochrane Library. The main final indicators were overall survival (OS), disease-free survival (DFS), and progression-free survival (PFS). Results: A total of 26 studies entailing 4,506 cases were included for a meta-analysis. The results showed that HAIC could improve advanced HCC patients' OS (HR, 0.49; 95\% Cl: 0.37-0.61) and PFS (HR, 0.52; 95\% Cl: 0.36-0.68). Remarkably, compared with Japan (HR, 0.58) and Korea (HR, 0.54), for the unresectable HCC patients, the HAIC group achieved higher efficacy on OS than the control group in China (HR, 0.24). The resectable HCC patients, who received HAIC adjuvant chemotherapy, exhibited favorable prognosis for OS (HR, 0.58; 95\% Cl: 0.27-0.88) and DFS (HR,
\end{abstract}

0.49; 95\% Cl: 0.31-0.68). Conclusion: HAIC improved longterm survival for both resectable and unresectable HCC patients in comparison with other therapies. However, the clinical effect of HAIC needs to be ascertained by large-scale well-designed studies.

(c) 2021 S. Karger AG, Basel

\section{Introduction}

Hepatocellular carcinoma (HCC) ranks sixth among the most common neoplasms worldwide, and it is attributed to $>700,000$ deaths yearly $[1,2]$. In Eastern Asia (mainly in China, Korea, and Japan), HCC is associated with high incidence rates, malignancy, and rapid development [3]. Hepatic resection, liver transplantation, and local ablation are regarded as the common curative treatment approaches for the patients. However, for most patients, the tumor stage had already advanced by the time of their first diagnosis. Besides, they exhibit liver dysfunction, extrahepatic metastasis, or macro/microscopic vascular invasion [4]. The treatment for advanced HCC pa-

Shengzhou Li and Jiaxuan Xu contributed equally to this work. karger@karger.com www.karger.com/che
(C) 2021 S. Karger AG, Basel
Correspondence to:

Dingcheng Zheng, dingcheng1984@163.com$$
\text { 监 }
$$ \\ Karger"}


tients is focused on transcatheter arterial chemoembolization (TACE), yttrium-90 radioembolization, sorafenib, and immunotherapy. Nevertheless, the overall therapeutic effect is limited [5-8]. The overall prognosis of the patients undergoing liver resections is normally poor as a result of the high postoperative recurrence rate [9].

A novel regimen exhibiting great safety as well as efficacy is urgently necessary to prolong the survival rate of the HCC patients. Recently, hepatic arterial infusion chemotherapy (HAIC) has been carried out as a new palliative chemotherapy for treating the advanced HCC patients [10-12]. Besides, it is an appropriate adjuvant chemotherapy for the resectable HCC patients following hepatic resection [13-15]. The HAIC procedure made chemotherapeutic agents to be delivered directly into the feeding arteries of the liver tumors, at high local drug concentrations using an implantable port system. As such, it ensured antitumor efficacy and minimized systemic toxicity, due to the stronger first-pass effect in comparison with the systemic chemotherapy [16]. Previous literature had assessed the effect of HAIC in treatment of the unresectable/resectable HCC patients in the palliative chemotherapy as well as adjuvant chemotherapy. However, HAIC efficacy and safety findings differed exceptionally and have been controversial [17]. As such, the main objective of this meta-analysis was to evaluate the outcomes of the long-term prognosis of HCC patients exhaustively using HAIC in the palliative chemotherapy and adjuvant chemotherapy.

\section{Materials and Methods}

\section{Literature Search Strategy}

Meta-analysis was carried out with respect to the Preferred Reporting Items for Systematic Review and Meta-Analysis guidelines [18]. Published studies in the English language from the inception to July 2020 were searched in the following electronic databases: PubMed, Web of Science, Embase, and Cochrane Library. The article search strategies that were used included ("HAIC" OR "Hepatic Arterial Infusion Chemotherapy") AND ("Hepatocellular Carcinoma" OR "Liver Cell Carcinomas" OR "Hepatoma" OR "Adult Liver Cancer" OR "HCC"). The reference lists of the retrieved studies were reviewed manually to obtain eligible articles.

\section{Inclusion Criteria}

The inclusion criteria in the meta-analysis entailed (1) HCC patients subjected to palliative HAIC or adjuvant HAIC after hepatic resection in the intervention group, (2) the included literature should be the original study, including observational studies (OBSs) or randomized controlled trials (RCTs), and (3) at least OS, DFS, and PFS related to prognostic information should be reported.

Hepatic Arterial Infusion Chemotherapy

and Hepatocellular Carcinoma

\section{Exclusion Criteria}

The exclusion criteria in the meta-analysis included the following: (1) in case of duplicate publications, the latest or high-quality study was included, (2) HAIC as a regimen instead of the only variable, such as HAIC + TACE versus sorafenib, (3) relevant data were unavailable, and (4) the studies included secondary liver carcinoma.

\section{Data Extraction and Quality Evaluation}

Under both the inclusion and exclusion criteria, two authors independently reviewed the included studies and extracted the related information. Disagreements between the two investigators regarding data extraction were solved via discussion or third-party intervention. The extracted appropriate information included author, year of publication, country, and patients' demographic and clinicopathological characteristics such as etiology and extrahepatic metastasis as well as the long-term prognosis for OS, PFS, or DFS.

The Newcastle-Ottawa Scale (NOS) was used to assess the quality of the incorporated OBSs under three aspects: patient selection, comparability of the groups, and outcome evaluation. The literature scores $>6$ were considered to be of high quality [19]. The Cochrane Collaboration's tool was used to evaluate the potential risks of bias on the retrieved RCTs.

\section{Statistical Analysis}

The hazard ratio (HR) and 95\% confidence interval (CI) of each literature were used to establish the relationship between the therapies and prognosis. $I^{2}$ statistic was used to carry out the heterogeneity test. Since many regimens were included, the random-effect models were used to ensure greater reliability. Stata 12.0 software was used to conduct publication bias assessment and sensitivity analysis, when the number of the included literature is $\geq 10$. Publication bias was quantitatively assessed using the Begg's and Egger's test. Sensitivity analysis was used to determine the stability of the conclusions. The $p$ value $<0.05$ was considered statistically significant.

\section{Results}

\section{Data Collection and Characteristics}

With regard to the search strategy, a total of 4,512 records were initially produced from the electronic databases. After the extraction of the duplicates, 2,003 records remained altogether. Twenty-six studies $[5,8$, $10-15,20-37]$ totaling 4,506 cases were eventually included following a strict layered screening. The comprehensive study review and selection process is as shown in Figure 1.

Twenty-six studies comprising 6 RCTs and 20 OBSs originated from China, Japan, and Korea. Among them, 21 and 5 studies presented the role of HAIC in palliative and adjuvant chemotherapies, respectively. Concerning the unresectable HCC patients, either HAIC alone or HAIC + sorafenib was the common interventional meth- 


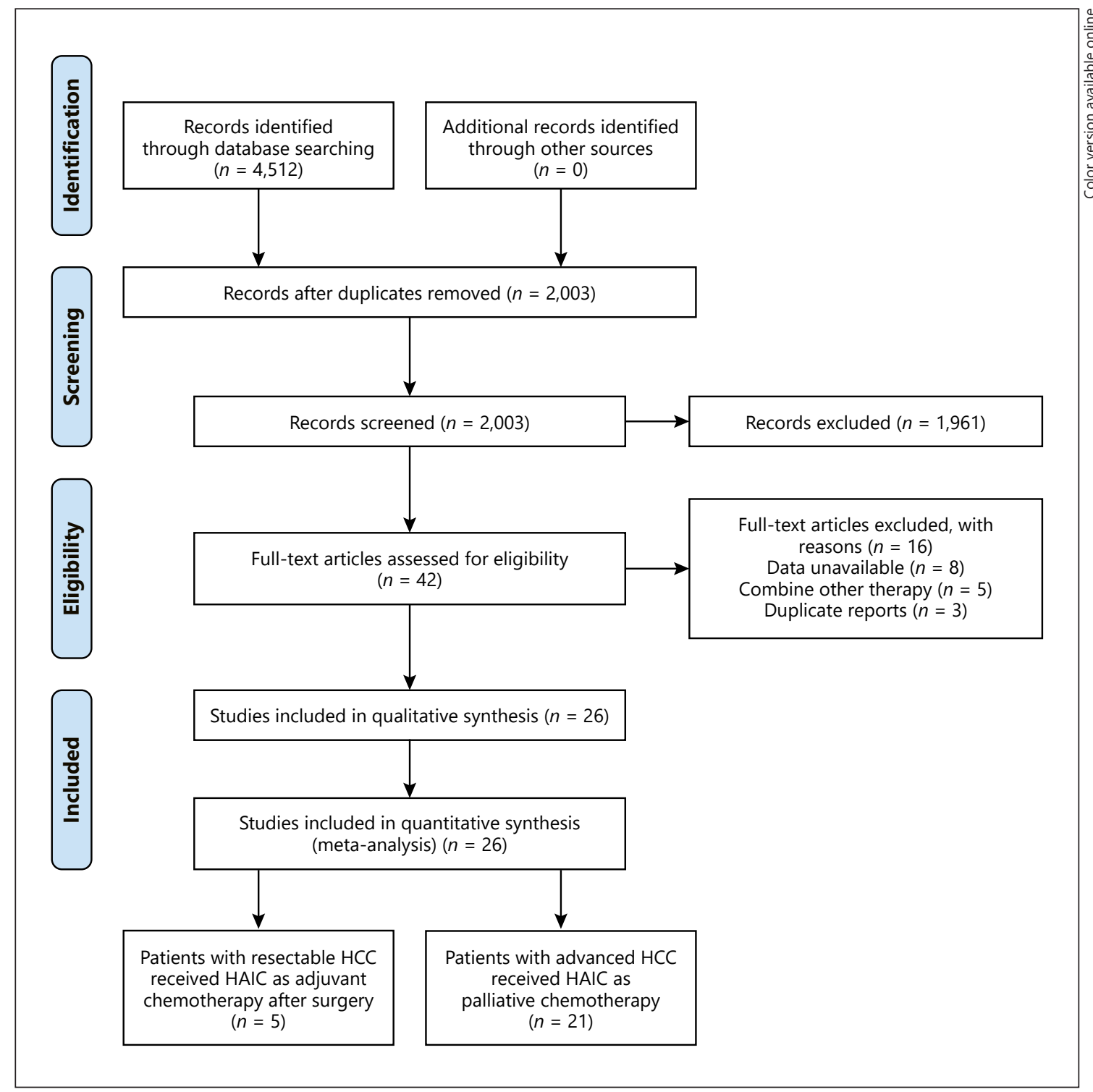

Fig. 1. A schematic flow for the selection of the articles included in this meta-analysis. HCC, hepatocellular carcinoma; HAIC, hepatic arterial infusion chemotherapy.

od. However, for the resectable HCC patients, HAIC was the adjuvant chemotherapy following surgery (Table 1). Besides, other information such as the status of extrahepatic metastasis, previous treatment, and hepatitis B virus (HBV) and hepatitis $\mathrm{C}$ virus infections was also recorded (online suppl. Table 1; for all online suppl. material, see www.karger.com/doi/10.1159/000518257).

The outcomes of OBS assessment indicated that the incorporated studies were of high quality. However, in the RCTs, personnel and patients were not blinded, and other biases were not mentioned (online suppl. Table 2).

\section{Efficacy of HAIC on Palliative Treatment of}

Unresectable HCC

The random-effects model showed that the meta-analysis of the 21 studies of palliative chemotherapy had potential OS advantage for the unresectable HCC patients in the HAIC group unlike the control group (HR, 0.49 ; 95\% CI: 0.37-0.61) (Fig. 2). Besides, the heterogeneity was high. It was also noted that for the unresectable HCC patients, the HAIC group achieved higher efficacy on OS than the control group in China (HR, 0.24) in comparison with Japan (HR, 0.58) and Korea (HR, 0.54). In various regimens of 
Table 1. Characteristics of all the studies included in the meta-analysis

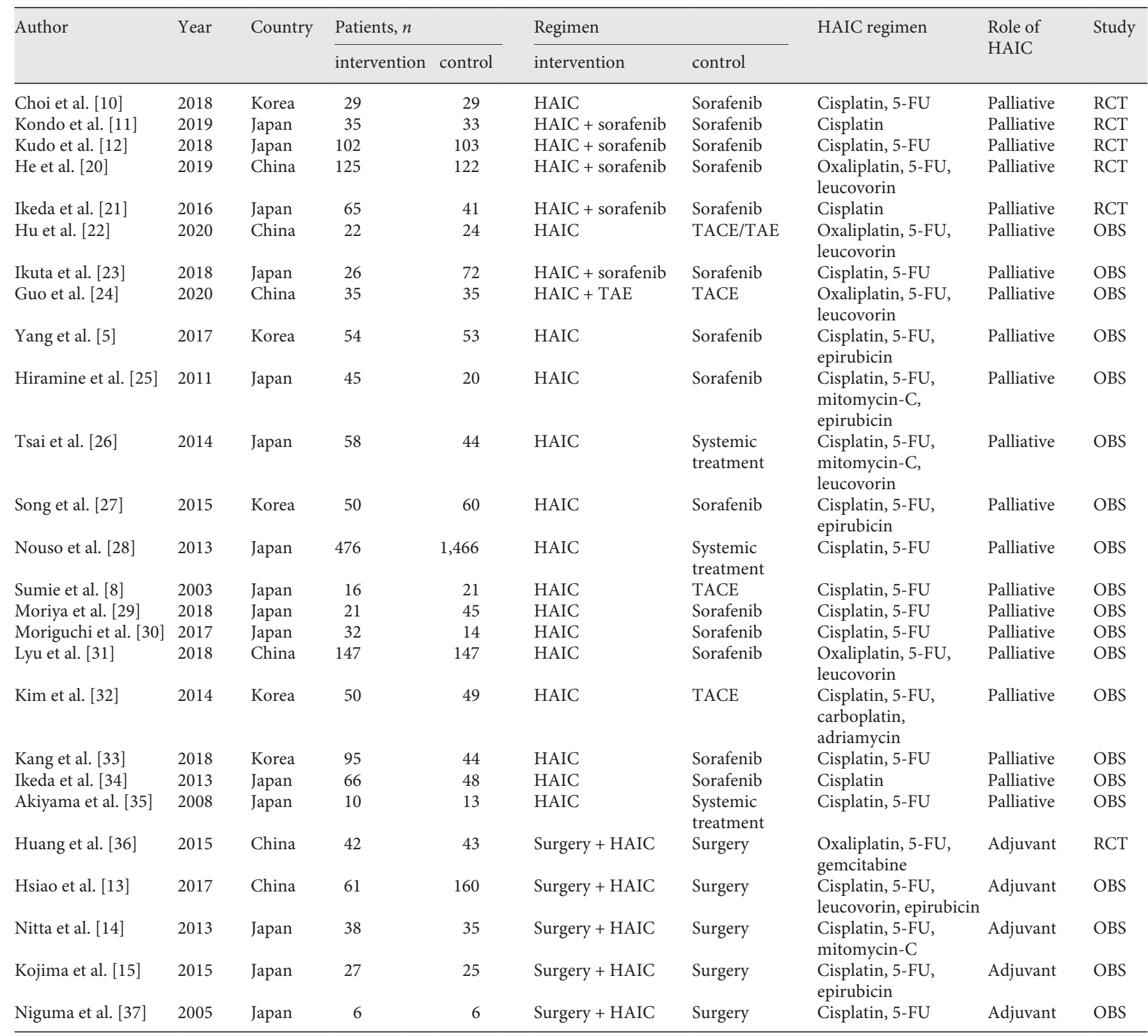

HAIC, hepatic artery infusion chemotherapy; RCT, randomized controlled trial; OBS, observational study; TACE, transcatheter arterial chemoembolization; TAE, transcatheter arterial embolization; 5-FU, 5-fluorouracil.

intergroup (HAIC vs. sorafenib, HAIC vs. TACE, HAIC vs. others, and HAIC + sorafenib vs. SORAFENIB), the patients receiving HAIC showed improved OS, and the results were statistically significant (Table 2).

Analysis on the 11 included studies referred to as PFS implied that HAIC could improve PFS of unresectable patients (HR, 0.52; 95\% CI: 0.36-0.68) (Fig. 3). Besides, subgroup analyses showed that in comparison with the control group, the HAIC group achieved the most benefit on PFS in China (HR, 0.29), followed by Korea (HR, 0.50). However, in Japan (HR, 0.98), the HAIC did not improve the patients' PFS. The HAIC group evidently increased the PFS (HR, 0.32) compared with the TACE regimen. However, HAIC efficacy was not statistically significant in comparison with sorafenib (HR, 0.64; 95\% CI: 0.26-1.03) (Table 2). 
Fig. 2. Forest plot of the overall survival of the patients with unresectable hepatocellular carcinoma on palliative hepatic arterial infusion chemotherapy $(p<0.001)$.

\begin{tabular}{|c|c|c|c|}
\hline Study ID & & $\mathrm{HR}(95 \% \mathrm{Cl})$ & $\begin{array}{l}\text { Weight, } \\
\%\end{array}$ \\
\hline Choi, 2018 & $\rightarrow$ & $0.32(0.15,0.71)$ & 5.21 \\
\hline Kondo, 2019 & $1 \rightarrow$ & $1.08(0.63,1.86)$ & 2.49 \\
\hline Kudo, 2018 & $\rightarrow$ & $0.99(0.73,1.35)$ & 4.91 \\
\hline $\mathrm{He}, 2019$ & - & $0.35(0.26,0.48)$ & 6.86 \\
\hline Ikeda, 2016 & - & $0.68(0.44,1.05)$ & 4.95 \\
\hline $\mathrm{Hu}, 2020$ & $\leftarrow$ & $0.17(0.06,0.51)$ & 5.79 \\
\hline Ikuta, 2018 & $\div$ & $0.52(0.30,0.92)$ & 4.90 \\
\hline Yang, 2017 & $\rightarrow$ & $0.47(0.30,0.75)$ & 5.78 \\
\hline Hiramine, 2011 & $\longrightarrow$ & $1.27(0.43,3.70)$ & 0.50 \\
\hline Hiramine, 2011 & $\rightarrow$ & $0.30(0.13,0.69)$ & 5.21 \\
\hline Tsai, 2014 & $\leftarrow$ & $0.32(0.20,0.51)$ & 6.47 \\
\hline Song, 2015 & $\rightarrow$ & $0.69(0.41,1.15)$ & 4.29 \\
\hline Nouso, 2013 & $\cdot$ & $0.48(0.41,0.56)$ & 7.08 \\
\hline Sumie, 2003 & 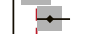 & $0.76(0.49,1.18)$ & 4.54 \\
\hline Moriya, 2018 & 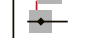 & $0.57(0.29,1.13)$ & 3.82 \\
\hline Moriguchi, 2017 & $\leftarrow$ & $0.25(0.09,0.67)$ & 5.15 \\
\hline Lyu, 2018 & -1 & $0.13(0.09,0.18)$ & 7.21 \\
\hline Kim, 2014 & - - & $0.68(0.42,1.08)$ & 4.69 \\
\hline Kang, 2018 & $\rightarrow$ & $0.66(0.43,1.01)$ & 5.11 \\
\hline Ikeda, 2013 & $T$ & $2.38(1.30,4.00)$ & 0.71 \\
\hline Akiyama, 2008 & 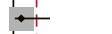 & $0.17(0.04,0.77)$ & 4.33 \\
\hline Overall $\left(R^{2}=86.7 \%, p=0.000\right)$ & $\phi$ & $0.49(0.37,0.61)$ & 100.00 \\
\hline-4 & 0 & & \\
\hline
\end{tabular}

Table 2. Subgroup analysis of the prognosis of HCC patients receiving HAIC

\begin{tabular}{lcccc}
\hline & Studies, & HR & $95 \%$ CI & $\begin{array}{l}\text { Heterogeneity } \\
\left(I^{2}\right), \%\end{array}$ \\
& $n$ & & & \\
& & & & \\
\hline Palliative chemotherapy & & & & \\
OS & 12 & 0.58 & $0.42-0.74$ & 67.3 \\
Japan & 4 & 0.24 & $0.10-0.38$ & 82.4 \\
China & 5 & 0.54 & $0.40-0.68$ & 13.3 \\
Korea & 10 & 0.45 & $0.25-0.65$ & 80.5 \\
HAIC versus sorafenib & 3 & 0.52 & $0.12-0.92$ & 81.8 \\
HAIC versus TACE & 3 & 0.38 & $0.22-0.54$ & 63.1 \\
HAIC versus others & 5 & 0.67 & $0.39-0.96$ & 81.2 \\
HAIC + sorafenib versus sorafenib & 16 & 0.43 & $0.30-0.57$ & 86.5 \\
HAIC versus sorafenib/TACE/others & & & & \\
PFS & 4 & 0.98 & $0.63-1.32$ & 63.0 \\
Japan & 4 & 0.29 & $0.14-0.43$ & 82.2 \\
China & 3 & 0.50 & $0.23-0.78$ & 51.8 \\
Korea & 4 & 0.64 & $0.26-1.03$ & 82.3 \\
HAIC versus sorafenib & 2 & 0.32 & $0.17-0.81$ & 89.6 \\
HAIC versus TACE & 4 & 0.68 & $0.34-1.01$ & 86.4 \\
HAIC + sorafenib versus sorafenib & 6 & 0.49 & $0.24-0.75$ & 87.6 \\
HAIC versus sorafenib/TACE/others & 5 & 0.58 & $0.34-0.83$ & 82.3 \\
HAIC + (sorafenib/TACE) versus sorafenib/TACE & & & & \\
Adjuvant chemotherapy & & & & \\
OS Japan & 3 & 0.43 & $0.08-0.78$ & 49.6 \\
China & 2 & 0.78 & $0.25-1.31$ & 74.4 \\
Japan & 2 & 0.51 & $0.28-0.74$ & 0.0 \\
\hline
\end{tabular}

HAIC, hepatic artery infusion chemotherapy; HCC, hepatocellular carcinoma; TACE, transcatheter arterial chemoembolization; OS, overall survival; PFS, progression-free survival; DFS, disease-free survival. 
Fig. 3. Forest plot of the progression-free survival of the patients with unresectable hepatocellular carcinoma on palliative hepatic arterial infusion chemotherapy $(p<$ $0.001)$.

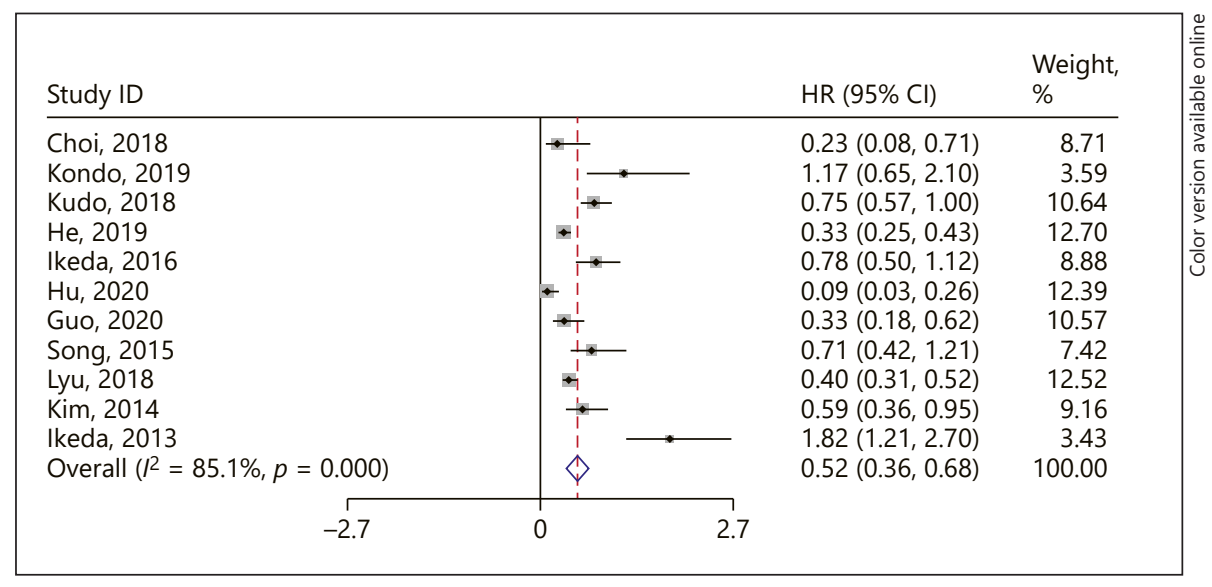

Fig. 4. Forest plot for the long-term survival of the patients with resectable hepatocellular carcinoma on adjuvant hepatic arterial infusion chemotherapy after hepatectomy (a OS, $p<0.001$; b DFS, $p<0.001$ ).

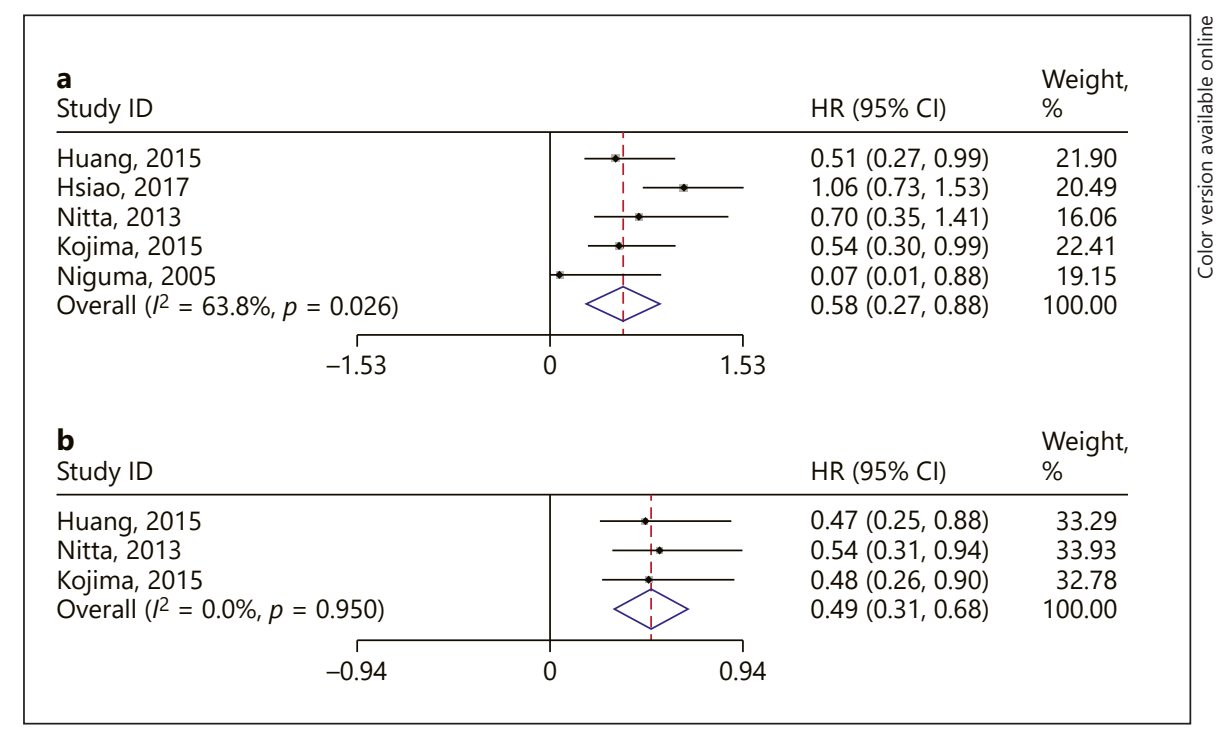

\section{Efficacy of HAIC on Adjuvant Chemotherapy of Resectable HCC}

HAIC was vital in adjuvant and palliative chemotherapies. Five and 3 studies entailed the OS and DFS, respectively, for the resectable HCC patients in the adjuvant chemotherapy. The HAIC group had a favorable prognosis on OS in comparison with the control group (HR, 0.58; 95\% CI: 0.27-0.88) and DFS (HR, 0.49; 95\% CI: 0.310.68 ) (Fig. 4). HAIC improved the Japanese patients' OS (HR, 0.43) and DFS (HR, 0.51). However, in China, the HAIC did not improve DFS (HR, 0.78; 95\% CI: 0.251.31) (Table 2).

\section{Sensitivity Analysis and Publication Bias}

Due to the limitation of the included literature of adjuvant chemotherapy, the sensitivity and publication bias tests were carried out for the included literature of palliative chemotherapy. The sensitivity analysis demonstrated a terrific stability of the HR for OS after sequentially omitting each included literature on palliative chemotherapy (online suppl. Fig. 1). The quantificational Egger's and Begg's tests were used to evaluate publication bias, and the result of Egger's and Begg's tests was not significantly different $(p>0.05)$. This suggested that there were no publication biases among the included literature on HAIC and OS (online suppl. Fig. 2).

Besides, the sensitivity analysis indicated that individual study had little impact on the final PFS results, and as such, the results remained stable (online suppl. Fig. 3). Similarly, no publication biases were realized in the included studies on HAIC and PFS ( $p>0.05)$ (online suppl. Fig. 4). 


\section{Discussion}

The findings of this meta-analysis suggested that HAIC improves OS and PFS of the advanced HCC patients unlike other therapies in the palliative chemotherapy. Prolonged OS and PFS was realized in the patients treated with HAIC, and this was consistent with the findings reported in previous studies [10-12]. In a randomized phase 3 trial [12], HAIC plus sorafenib combination therapy exhibited longer PFS in the advanced HCC patients in comparison with sorafenib monotherapy. This implies that combination therapy probably had additive anticancer effects on the patients. Remarkably, a comparative study [26], published in 2014, demonstrated that HAIC could achieve longer OS in patients suffering from advanced HCC than sorafenib treatment. However, the results were not statistically significant, which could have resulted from the status of most advanced HCC patients with extrahepatic spread and large tumor size $(>10 \mathrm{~cm})$. Besides, another OBS [35], originating from Japan, reported that HAIC improved OS significantly for the unresectable HCC patients in comparison with the systemic treatment (HR 0.17, $p=0.021$ ). However, the study size of the sample was small, only incorporating 23 patients, and this made the findings unconvincing.

To comprehensively explain the mechanism of HAIC affecting HCC, from the previous literature, we found out that the mechanism probably involves inhibition of the tumor cell proliferation and induction of tumor cell apoptosis [38-42]. Through HAIC, the chemotherapeutic drugs are delivered directly into the hepatic arteries of the liver, at high local drug concentrations. This improves the efficacy of the drug on malignant cells as much as possible. Meanwhile, with a first-pass effect of the hepatic clearance, the drugs are generally metabolized in the liver, resulting in low drug concentrations in the peripheral blood besides reducing the side effects [16]. 5-Fluorouracil (5-FU), a chemotherapeutical drug used in HAIC, prevents tumor cell proliferation via antimetabolism. With high local drug concentrations, HAIC enhances the mechanism by which 5-FU inhibits enzyme thymidylate synthase, thus preventing the thymidine formation demanded for DNA synthesis [38]. Besides, cisplatin, another chemotherapeutic agent used in HAIC, can cross connect with adenine and cytosine in the DNA, hence destroying the structure and function of DNA. It also interferes with the synthesis and repair mechanisms of DNA and consequently induces the apoptosis of the cancerous cells [39]. With regard to oxaliplatin, normally, it exerts its antitumor effect via inhibiting the replication and transcription of DNA [40,41]. Recent studies have reported that oxaliplatin can not only bind to a variety of subcellular structures but also act on tumor microenvironment and enhance the immune response to the tumor cells $[41,42]$.

This meta-analysis showed that HAIC could improve OS and PFS of the advanced HCC patients unlike other therapies. Although the heterogeneity was high, we performed the subgroup analysis to make the results more convincing and scientific. Among the different countries (China, Japan, and Korea), compared with the control group, HAIC improved the advanced HCC patients' OS and PFS. In China, the HAIC group exhibited the highest benefit for the patients than the control group (HR, 0.24). For Japan (HR, 0.58) and Korea (HR, 0.54), the results were also statistically significant. We realized that the selection of chemotherapeutic agents in HAIC treatment is an important factor in the prognosis of advanced HCC patients. In the previous research, cisplatin-based HAIC treatment had been applied in the unresectable HCC patients. However, the therapeutic program effect was unsatisfactory. In comparison with cisplatin, oxaliplatin had better biochemical, immunologic, and pharmacokinetic effect $[43,44]$. As such, it is better to use oxaliplatin instead of cisplatin in the HAIC scheme. In the included studies for the Chinese patients, most HAIC regimens adopted HAIC of oxaliplatin, leucovorin, and fluorouracil. On the other hand, Japan and Korea generally adopted HAIC treatment based on cisplatin for the advanced HCC patients [20, 22, 24, 26, 31]. We also realized that the average age of patients in China was lower than that in Japan and Korea. Many elderly patients were exceedingly pessimistic once they were diagnosed unlike the young patients who faced the disease with a positive mood. In addition, the elder patients' emotion and psychological status had a negative impact on the HCC development. During the clinical diagnosis and subsequent treatment, the elderly patients easily exhibited a variety of psychological problems including anxiety, tense, depression, and other aftermaths. This in turn reduced the treatment effect of the HCC greatly [45]. Also, a study found that the HBV-related HCC patients had benefited more from the treatment in comparison with non-HBV patients [23]. HBV infection is the major etiology of HCC patients in China.

With regard to the PFS subgroup analysis, we realized that the patients receiving HAIC did not improve PFS compared to the control group in Japan (HR, 0.98). A potential risk factor for the disease is chronic stress. In Japan, under a long-term chronic stress, the cortisol secre- 
tion level of HCC patients increased, which probably decreased the expression of the cortisol receptor. This in turn reduces the anti-inflammatory reaction thus promoting inflammation [46]. HCC is a malignant tumor that is closely associated with inflammation, which contributes to the occurrence and metastasis of HCC [47].

As an adjuvant chemotherapy, HAIC also plays a vital role in patients subjected to liver resection. The outcome of the subgroup analysis demonstrated that the Japanese patients receiving adjuvant HAIC regimen had longer OS and PFS than those who underwent liver resection only. In China, the HAIC regimen was not effective on the patients. The main reason behind this difference is probably the small number of the included literatures.

Before we conducted this study, we realized that some researchers had already carried out relevant meta-analyses. However, due to the lack of some related data and in-depth research, the studies had certain limitations [48-50]. Zhuang et al. [48] suggested that in comparison with sorafenib treatment, HAIC enhanced advanced HCC patients' long-term survival for OS (HR, 0.60) and PFS (HR, 0.69 ). However, the regimens only included HAIC and sorafenib, and this was therefore not appropriate for a comprehensive and systematic evaluation of the effect of HAIC on advanced HCC patients. Another limitation was that all of the included studies in the meta-analysis were OBSs. Ouyang et al. [49] suggested that sorafenib plus HAIC regimens showed significant improvement on OS (HR, 0.59) than sorafenib monotherapy. In addition, there were no statistics on the patient's PFS data, and the number of the included studies was only 5 , which notably decreased the quality of evidence. Long et al. [50] reported that HAIC improved HCC patients' OS (HR, 0.61) and DFS (HR, 0.66 ) in comparison with other therapies. However, an evident limitation was uncertainty in patient selection, and patients with various liver malignant tumors involving primary HCC and secondary HCC were included.

This study had several limitations as follows: first, most of the included articles in this study were OBSs, and a few articles were RCTs. This might have led to selection bias of the results and as such inevitably caused overvaluation or undervaluation of the measured effects. Second, due to the lack of relevant data on HCC patients, we could not perform more subgroup analyses in terms of age, HAIC regimen, and etiology, so as to explore the effects of HAIC in the specific research patients. Third, all of the populations evaluated in this meta-analysis were from Asian countries. For this reason, this conclusion could not be directly applied to the Western or African population.

Hepatic Arterial Infusion Chemotherapy

and Hepatocellular Carcinoma

\section{Conclusion}

Our study demonstrated that HAIC improved longterm survival in OS and PFS unlike other conventional therapies in patients suffering from advanced HCC. This was particularly observed among the patients in China. Besides, the combination of hepatic resection with adjuvant HAIC improved OS and DFS for patients suffering from resectable HCC. As such, HAIC is an alternative therapeutic choice for both resectable and unresectable HCC patients. However, large-scale prospective RCTs should be designed to ascertain the clinical effect of HAIC.

\section{Conflict of Interest Statement}

The authors declare that they have no conflicts of interest.

\section{Funding Sources}

The authors have no financial support to declare.

\section{Author Contributions}

Each author contributed significantly to the concept and development of the present manuscript. D.C.Z. and S.Z.L. designed the research process. J.X.X. and J.Z.H. searched the database for corresponding articles. H.Y.Z. and Y.X.S. extracted useful information from the articles above. T.Y. used statistical software for analysis. Y.J.H. drafted the meta-analysis. D.M.N. polished this article. All authors had read and approved the manuscript and ensured that this was the case.

References

1 Ferlay J, Shin HR, Bray F, Forman D, Mathers C, Parkin DM. Estimates of worldwide burden of cancer in 2008: GLOBOCAN 2008. Int J Cancer. 2010;127(12):2893-917.

2 Bosch FX, Ribes J, Díaz M, Cléries R. Primary liver cancer: worldwide incidence and trends. Gastroenterology. 2004;127(5 Suppl 1):S5-s16.

3 Tang A, Hallouch O, Chernyak V, Kamaya A, Sirlin CB. Epidemiology of hepatocellular carcinoma: target population for surveillance and diagnosis. Abdom Radiol. 2018;43(1):13-25.

4 Llovet JM, Bruix J. Systematic review of randomized trials for unresectable hepatocellular carcinoma: chemoembolization improves survival. Hepatology. 2003;37(2):429-42.

5 Yang H, Woo HY, Lee SK, Han JW, Jang B, Nam HC, et al. A comparative study of sorafenib and metronomic chemotherapy for Barcelona clinic liver cancer-stage $\mathrm{C}$ hepatocellular carcinoma with poor liver function. Clin Mol Hepatol. 2017;23(2):128-37. 
6 Floridi C, Pesapane F, Angileri SA, De Palma D, Fontana F, Caspani F, et al. Yttrium-90 radioembolization treatment for unresectable hepatocellular carcinoma: a single-centre prognostic factors analysis. Med Oncol. 2017; 34(10): 174.

7 Xing M, Kokabi N, Camacho JC, Kim HS. Prospective longitudinal quality of life and survival outcomes in patients with advanced infiltrative hepatocellular carcinoma and portal vein thrombosis treated with Yttrium-90 radioembolization. BMC Cancer. 2018;18(1): 75.

8 Sumie S, Yamashita F, Ando E, Tanaka M, Yano Y, Fukumori K, et al. Interventional radiology for advanced hepatocellular carcinoma: comparison of hepatic artery infusion chemotherapy and transcatheter arterial lipiodol chemoembolization. Am J Roentgenol. 2003;181(5):1327-34.

9 Takenaka K, Kawahara N, Yamamoto K, Kajiyama K, Maeda T, Itasaka $\mathrm{H}$, et al. Results of 280 liver resections for hepatocellular carcinoma. Arch Surg. 1996;131(1):71-6.

10 Choi JH, Chung WJ, Bae SH, Song DS, Song MJ, Kim YS, et al. Randomized, prospective, comparative study on the effects and safety of sorafenib versus hepatic arterial infusion chemotherapy in patients with advanced hepatocellular carcinoma with portal vein tumor thrombosis. Cancer Chemother Pharmacol. 2018;82(3):469-78.

11 Kondo M, Morimoto M, Kobayashi S, Ohkawa S, Hidaka H, Nakazawa T, et al. Randomized, phase II trial of sequential hepatic arterial infusion chemotherapy and sorafenib versus sorafenib alone as initial therapy for advanced hepatocellular carcinoma: SCOOP-2 trial. BMC Cancer. 2019;19(1): 954.

12 Kudo M, Ueshima K, Yokosuka O, Ogasawara S, Obi S, Izumi N, et al. Sorafenib plus low-dose cisplatin and fluorouracil hepatic arterial infusion chemotherapy versus sorafenib alone in patients with advanced hepatocellular carcinoma (SILIUS): a randomised, open label, phase 3 trial. Lancet Gastroenterol Hepatol. 2018;3(6):424-32.

13 Hsiao JH, Tsai CC, Liang TJ, Chiang CL, Liang HL, Chen IS, et al. Adjuvant hepatic arterial infusion chemotherapy is beneficial for selective patients with Hepatocellular carcinoma undergoing surgical treatment. Int J Surg. 2017;45:35-41.

14 Nitta H, Beppu T, Imai K, Hayashi H, Chikamoto A, Baba H. Adjuvant hepatic arterial infusion chemotherapy after hepatic resection of hepatocellular carcinoma with macroscopic vascular invasion. World J Surg. 2013; 37(5):1034-42.

15 Kojima H, Hatano E, Taura K, Seo S, Yasuchika $\mathrm{K}$, Uemoto S. Hepatic resection for hepatocellular carcinoma with tumor thrombus in the major portal vein. Dig Surg. 2015;32(6):413-20.

16 Song MJ. Hepatic artery infusion chemotherapy for advanced hepatocellular carcinoma. World J Gastroenterol. 2015;21(13):3843-9.
17 Bruix J, Sherman M. Management of hepatocellular carcinoma: an update. Hepatology. 2011;53(3):1020-2.

18 Moher D, Liberati A, Tetzlaff J, Altman DG. Preferred reporting items for systematic reviews and meta-analyses: the PRISMA statement. BMJ. 2009;339:b2535.

19 Stang A. Critical evaluation of the NewcastleOttawa scale for the assessment of the quality of nonrandomized studies in meta-analyses. Eur J Epidemiol. 2010;25(9):603-5.

20 He M, Li Q, Zou R, Shen J, Fang W, Tan G, et al. Sorafenib plus hepatic arterial infusion of oxaliplatin, fluorouracil, and leucovorin versus sorafenib alone for hepatocellular carcinoma with portal vein invasion: a randomized clinical trial. JAMA Oncol. 2019;5(7): 953-60.

21 Ikeda M, Shimizu S, Sato T, Morimoto M, Kojima Y, Inaba $Y$, et al. Sorafenib plus hepatic arterial infusion chemotherapy with cisplatin versus sorafenib for advanced hepatocellular carcinoma: randomized phase II trial. Ann Oncol. 2016;27(11):2090-6.

$22 \mathrm{Hu}$ J, Bao Q, Cao G, Zhu X, Yang R, Ji X, et al. Hepatic arterial infusion chemotherapy using oxaliplatin plus 5-Fluorouracil versus transarterial chemoembolization/embolization for the treatment of advanced hepatocellular carcinoma with major portal vein tumor thrombosis. Cardiovasc Intervent Radiol. 2020; 43(7):996-1005.

23 Ikuta S, Aihara T, Yamanaka N. Efficacy of sequential sorafenib plus hepatic arterial infusion chemotherapy in patients with Barcelona clinic liver cancer stage B and C hepatocellular carcinoma: a retrospective single-institution study. Contemp Oncol. 2018;22(3):16571.

24 Guo W, Gao J, Zhuang W, Wu Z, Li B, Chen S. Efficacy and safety of hepatic arterial infusion chemotherapy combined with transarterial embolization for unresectable hepatocellular carcinoma: a propensity score-matching cohort study. JGH Open. 2020;4(3):477-83.

25 Hiramine $Y$, Uto $H$, Imamura Y, Tabu K, Baba Y, Hiwaki T, et al. Sorafenib and hepatic arterial infusion chemotherapy for unresectable advanced hepatocellular carcinoma: a comparative study. Exp Ther Med. 2011;2(3): 433-41.

26 Tsai WL, Lai KH, Liang HL, Hsu PI, Chan $\mathrm{HH}$, Chen WC, et al. Hepatic arterial infusion chemotherapy for patients with huge unresectable hepatocellular carcinoma. PLoS One. 2014;9(5):e92784.

27 Song DS, Song MJ, Bae SH, Chung WJ, Jang JY, Kim YS, et al. A comparative study between sorafenib and hepatic arterial infusion chemotherapy for advanced hepatocellular carcinoma with portal vein tumor thrombosis. J Gastroenterol. 2015;50(4): 445-54.

28 Nouso K, Miyahara K, Uchida D, Kuwaki K, Izumi N, Omata $\mathrm{M}$, et al. Effect of hepatic arterial infusion chemotherapy of 5-fluorouracil and cisplatin for advanced hepatocellular carcinoma in the nationwide survey of primary liver cancer in Japan. Br J Cancer. 2013; 109(7):1904-7.

29 Moriya K, Namisaki T, Sato S, Douhara A, Furukawa M, Kawaratani H, et al. Efficacy of bimonthly hepatic arterial infusion chemotherapy for advanced hepatocellular carcinoma. J Gastrointest Oncol. 2018;9(4):741-9.

30 Moriguchi $M$, Aramaki T, Nishiofuku $\mathrm{H}$, Sato R, Asakura K, Yamaguchi K, et al. Sorafenib versus hepatic arterial infusion chemotherapy as initial treatment for hepatocellular carcinoma with advanced portal vein tumor thrombosis. Liver Cancer. 2017; 6(4):275-86.

31 Lyu N, Kong Y, Mu L, Lin Y, Li J, Liu Y, et al. Hepatic arterial infusion of oxaliplatin plus fluorouracil/leucovorin versus sorafenib for advanced hepatocellular carcinoma. J Hepatol. 2018;69(1):60-9.

32 Kim KH, Kim MS, Chang JS, Han KH, Kim DY, Seong J. Therapeutic benefit of radiotherapy in huge $(\geq 10 \mathrm{~cm})$ unresectable hepatocellular carcinoma. Liver Int. 2014;34(5):78494.

33 Kang MK, Park JG, Lee HJ. Comparison of clinical outcomes between sorafenib and hepatic artery infusion chemotherapy in advanced hepatocellular carcinoma: a strobecompliant article. Medicine. 2018;97(17): e0611.

34 Ikeda M, Mitsunaga S, Shimizu S, Ohno I, Takahashi H, Okuyama H, et al. Efficacy of sorafenib in patients with hepatocellular carcinoma refractory to transcatheter arterial chemoembolization. J Gastroenterol. 2014; 49(5):932-40.

35 Akiyama M, Miyaaki H, Miuma S, Shibata H, Fujimoto M, Takeshita S, et al. Significance of trans-hepatic arterial chemotherapy for advanced hepatocellular carcinoma with portal vein tumor thrombus. Oncol Rep. 2008;20(2): 353-7.

36 Huang SX, Wu YL, Tang CW, Feng WM, Xu YQ, Bao Y, et al. Prophylactic hepatic artery infusion chemotherapy improved survival after curative resection in patients with hepatocellular carcinoma. Hepatogastroenterology. 2015;62(137):122-5.

37 Niguma T, Mimura T, Tutui N. Adjuvant arterial infusion chemotherapy after resection of hepatocellular carcinoma with portal thrombosis: a pilot study. J Hepatobiliary Pancreat Surg. 2005;12(3):249-53.

38 Wigmore PM, Mustafa S, El-Beltagy M, Lyons L, Umka J, Bennett G. Effects of 5-FU. Adv Exp Med Biol. 2010;678:157-64.

39 Dasari S, Tchounwou PB. Cisplatin in cancer therapy: molecular mechanisms of action. Eur J Pharmacol. 2014;740:364-78.

40 Kelland L. The resurgence of platinum-based cancer chemotherapy. Nat Rev Cancer. 2007; 7(8):573-84.

41 Hato SV, Khong A, de Vries IJ, Lesterhuis WJ. Molecular pathways: the immunogenic effects of platinum-based chemotherapeutics. Clin Cancer Res. 2014;20(11):2831-7. 
42 Gatti L, Cassinelli G, Zaffaroni N, Lanzi C, Perego P. New mechanisms for old drugs: Insights into DNA-unrelated effects of platinum compounds and drug resistance determinants. Drug Resist Updat. 2015;20:1-11.

43 Bruno PM, Liu Y, Park GY, Murai J, Koch CE, Eisen TJ, et al. A subset of platinum-containing chemotherapeutic agents kills cells by inducing ribosome biogenesis stress. Nat Med. 2017;23(4):461-71.

44 Tesniere A, Schlemmer F, Boige V, Kepp O, Martins I, Ghiringhelli F, et al. Immunogenic death of colon cancer cells treated with oxaliplatin. Oncogene. 2010;29(4):482-91.
45 Hansen L, Dieckmann NF, Kolbeck KJ, Naugler WE, Chang MF. Symptom distress in patients with hepatocellular carcinoma toward the end of life. Oncol Nurs Forum. 2017; 44(6):665-73.

46 Zhang W, Wang X, Huang R, Jin K, Zhangyuan G, Yu W, et al. Prognostic value of marital status on stage at diagnosis in hepatocellular carcinoma. Sci Rep. 2017;7:41695.

47 Marx J. Cancer research. Inflammation and cancer: the link grows stronger. Science. 2004; 306(5698):966-8.
48 Zhuang BW, Li W, Xie XH, Hu HT, Lu MD, Xie XY. Sorafenib versus hepatic arterial infusion chemotherapy for advanced hepatocellular carcinoma: a systematic review and metaanalysis. Jpn J Clin Oncol. 2019;49(9):845-55.

49 Ouyang G, Pan G, Xu H, Wu Y, Liu Z, Lu W, et al. Sorafenib plus hepatic arterial infusion chemotherapy in advanced hepatocellular carcinoma: a systematic review and meta-analysis. J Clin Gastroenterol. 2020;54(8):675-81.

50 Long GB, Xiao CW, Zhao XY, Zhang J, Li X. Effects of hepatic arterial infusion chemotherapy in the treatment of hepatocellular carcinoma: a meta-analysis. Medicine. 2020; 99(26):e20745. 